\title{
Melanoma cell adhesion molecule as an emerging biomarker with prognostic significance in systolic heart failure
}

\begin{abstract}
Background: Melanoma cell adhesion molecule (MCAM) is a marker of endothelial damage. MCAM diagnostic and prognostic value was assessed in chronic heart failure (CHF). Materials \& methods: $130 \mathrm{CHF}$ patients and 32 controls were included in the study. Telephone follow-up lasted one year. End points were: death from all causes, and hospitalization with CHF exacerbation. Results: MCAM was higher in patients than in controls $(p=0.01)$. Receiver operator curve analysis revealed that MCAM may serve as a predictor of death (area under the curve: $0.8404 ; p<0.002$ ). Patients with MCAM above $500 \mathrm{ng} / \mathrm{ml}$ had worse prognosis $(p=0.03$ ). NT-proBNP and age were independent predictors of death in multivariate analysis. Conclusion: The increased MCAM indicates endothelial damage in CHF and may serve as a marker of worse prognosis in these patients.
\end{abstract}

First draft submitted: 28 February 2016; Accepted for publication: 22 April 2016; Published online: 29 June 2016

Keywords: chronic heart failure $\bullet$ melanoma cell adhesion molecule $\bullet$ prognosis

Melanoma cell adhesion molecule (MCAM), also known as cluster of differentiation molecule 146 (CD 146), is a glycoprotein belonging to the immunoglobulin superfamily. MCAM was first observed on malignant melanoma cells, specifically those within metastatic lesions [1]. It is mostly expressed on a cell surface, however, it has also been found within cytoplasm and at the cell-cell junctions $[2,3]$. In normal human tissues the expression of MCAM is limited mainly to endothelium, where it is concentrated at the intercellular junctions [4], but it may also be encountered on smooth muscle cells, neurons and in embryonal tissues [5]. MCAM, with its constitutional expression on endothelium, is also widely used to detect and isolate circulating endothelial cells (CEC), which serve as a marker of endothelial damage, observed in numerous pathological conditions including inflammatory $[6,7]$, infectious $[8]$, neo- plastic [9] and cardiovascular disorders [10,11]. Heart failure was found to be associated with increased amount of CEC reflecting extensive endothelial damage [12]. Some authors suggest that endothelial abnormalities encountered in heart failure patients may contribute to the elevated thrombotic risk and influence prognosis in this population [13]. Currently used methods of CEC quantification based on immunomagnetic isolation, flow cytometry or fluorescence-activated cell sorting are technically complex, expensive and time consuming. It has been reported that MCAM in its soluble form found in plasma serves as a surrogate marker of circulating endothelial cells [14]. The expression of MCAM on circulating endothelial cells and the observed correlation between its plasma concentration and conservatively measured CEC led to the assumption that soluble MCAM may be used as the equivalent of CEC. We
Joanna Banach*,1, Magdalena Grochowska', Lidia Gackowska², Katarzyna Buszko3, Robert Bujak', Wojciech Gilewski', Izabela Kubiszewska², Łukasz Wołowiec', Jacek Michałkiewicz ${ }^{2}$ \& Władysław Sinkiewicz ${ }^{1}$

'IInd Clinic of Cardiology, Colegium Medicum of Nicolaus Copernicus University in Bydgoszcz, University Hospital nr 2 in Bydgoszcz, Poland ${ }^{2}$ Department of Immunology, Collegium Medicum of Nicolaus Copernicus University (NCU) in Bydgoszcz, Poland ${ }^{3}$ Department of Theoretical Foundations of Biomedical Sciences \& Medical Informatics, Colegium Medicum of Nicolaus Copernicus University in Bydgoszcz, Poland *Author for correspondence: Tel.:/Fax: +48523655653 joannna@op.pl

\section{Future : Medicine part of}


Research Article Banach, Grochowska, Gackowska et al.

\begin{tabular}{|c|c|c|c|c|c|}
\hline \multicolumn{5}{|l|}{ Clinical characteristic } & \multirow[t]{2}{*}{ p-value } \\
\hline & All & Stable & Exacerbated & Controls & \\
\hline$n$ & 130 & 65 & 65 & 32 & - \\
\hline Males & 107 & 55 & 52 & 22 & - \\
\hline Age (mean $\pm S D)$; years & $59.8 \pm 13.1$ & $57.3 \pm 9.8$ & $61.5 \pm 14.1$ & $54.1 \pm 4.1$ & NS \\
\hline BMI $\left(\right.$ mean \pm SD) $; \mathrm{kg} / \mathrm{m}^{2}$ & $28.7 \pm 5.8$ & $28.2 \pm 5.6$ & $28.6 \pm 6.0$ & $26.7 \pm 2.4$ & NS \\
\hline Ischemic etiology, n (\%) & $66(53 \%)$ & $32(49 \%)$ & $34(56 \%)$ & NA & NS \\
\hline \multirow[t]{2}{*}{ NYHA II/III/IV, n (\%) } & $39 \% / 51 \% / 9 \%$ & $55 \% / 45 \% / 0 \%$ & $24 \% / 57 \% / 18 \%$ & \multirow[t]{2}{*}{ NA } & \multirow[t]{2}{*}{-} \\
\hline & $52 / 66 / 12$ & $36 / 29 / 0$ & $16 / 37 / 12$ & & \\
\hline Peripheral edema, n (\%) & $40(31 \%)$ & $8(12 \%)$ & $32(49 \%)$ & NA & $<0.05^{\ddagger}$ \\
\hline Pulmonary congestion, $\mathrm{n}(\%)$ & $37(28 \%)$ & $5(8 \%)$ & $32(49 \%)$ & NA & $<0.05^{\ddagger}$ \\
\hline LVEF $($ mean \pm SD); \% & $27 \pm 8$ & $27 \pm 7$ & $26 \pm 8$ & $67 \pm 5$ & $<0.001^{\dagger}$ \\
\hline Sinus rhythm, n (\%) & $97(75 \%)$ & $50(76 \%)$ & $47(72 \%)$ & 32 & $<0.001^{\dagger}$ \\
\hline DM, n (\%) & $47(36 \%)$ & $21(32 \%)$ & $26(40 \%)$ & 0 & $<0.001^{\dagger}$ \\
\hline Hypertension, n (\%) & $66(53 \%)$ & $33(50 \%)$ & $33(50 \%)$ & 0 & $<0.001^{\dagger}$ \\
\hline Hyperlipidemia, n (\%) & $96(74 \%)$ & $47(72 \%)$ & $49(75 \%)$ & 0 & $<0.001^{\dagger}$ \\
\hline Smoking, n (\%) & $37(28)$ & $19(25 \%)$ & $18(24 \%)$ & 0 & $<0.001^{\dagger}$ \\
\hline Death, n (\%) & $14(11 \%)$ & $5(8)$ & $9(14)$ & 0 & $<0.001^{\dagger}$ \\
\hline Hospitalization, n (\%) & $60(46 \%)$ & $33(51 \%)$ & $27(41 \%)$ & NA & $<0.001^{\dagger}$ \\
\hline \multicolumn{6}{|l|}{ Laboratory Tests $^{\dagger}$} \\
\hline $\mathrm{Hb}(\mathrm{g} / \mathrm{dl})$ & $13.9 \pm 1.5$ & $14.1 \pm 0.9$ & $13.7 \pm 1.9$ & $14.1 \pm 2.1$ & NS \\
\hline CRP (mg/l) & $5.8 \pm 6.6$ & $6.1 \pm 8.9$ & $5.9 \pm 4.7$ & $1.2 \pm 1.2$ & $<0.0001^{\dagger}$ \\
\hline Nt-proBNP (pg/ml) & $1862 \pm 5957$ & $1643.92 \pm 1776$ & $5142 \pm 7391$ & Not done & $<0.0001^{\ddagger}$ \\
\hline Troponin T $(\mu \mathrm{g} / \mathrm{l})$ & $0.02 \pm 0.03$ & $0.03 \pm 0.03$ & $0.03 \pm 0.02$ & Not done & $\mathrm{NS}^{\ddagger}$ \\
\hline GFR (ml/min) & 68 & 62 & 65 & 92 & $<0.001^{\dagger}$ \\
\hline \multicolumn{6}{|l|}{ Treatment } \\
\hline ACEI, n (\%) & $105(82 \%)$ & $51(78 \%)$ & $54(83)$ & NA & $\mathrm{NS}^{\ddagger}$ \\
\hline$A R B, n(\%)$ & $23(18 \%)$ & $14(22 \%)$ & $9(14 \%)$ & NA & $\mathrm{NS}^{\ddagger}$ \\
\hline Betablockers, n (\%) & $129(99 \%)$ & $65(100 \%)$ & $64(99 \%)$ & NA & $\mathrm{NS}^{\ddagger}$ \\
\hline Statin, n (\%) & $105(81 \%)$ & $58(89 \%)$ & $47(72 \%)$ & NA & $\mathrm{NS}^{\ddagger}$ \\
\hline ASA, n (\%) & $61(47 \%)$ & $26(40 \%)$ & $35(54 \%)$ & NA & $\mathrm{NS}^{\ddagger}$ \\
\hline Digoxin, $\mathrm{n}(\%)$ & $35(27 \%)$ & $22(34 \%)$ & $13(20 \%)$ & NA & $<0.05^{\ddagger}$ \\
\hline Spironolactone, n (\%) & $96(74 \%)$ & $45(69 \%)$ & $51(78 \%)$ & NA & $\mathrm{NS}^{\ddagger}$ \\
\hline Eplerenon, n (\%) & $23(18 \%)$ & $15(23 \%)$ & $8(12 \%)$ & NA & $<0.05^{\ddagger}$ \\
\hline Diuretic, n (\%) & $101(78 \%)$ & $36(55 \%)$ & $65(100 \%)$ & NA & $<0.05^{\ddagger}$ \\
\hline \multicolumn{6}{|c|}{$\begin{array}{l}{ }^{\dagger} \mathrm{p} \text {-value for the difference between controls and all patients. } \\
{ }^{\dagger} \mathrm{p} \text {-value for the difference between stable and exacerbated patients. } \\
\text { ACEl: Angiotensin converting enzyme inhibitors; ARB: Angiotensin receptor blockers; ASA: Acetylsalicilic acid; BMI: Body mass index; CRP: C-reactive protein; } \\
\text { DM: Diabetes mellitus; GFR: Glomerular filtration rate; Hb: Hemoglobin; LVEF: Left ventricular ejection fraction; NA: Not applicable; NS: Nonsignificant; NYHA: New } \\
\text { York Heart Association. }\end{array}$} \\
\hline
\end{tabular}

hypothesized that chronic heart failure with reduced ejection fraction will lead to the increase of MCAM plasma concentration, reflecting the increase in circulating endothelial cells amount, and that it would have unfavorable impact on prognosis.

\section{Aim of the study}

The aim of the study was to evaluate MCAM plasma concentration in patients with chronic heart failure with reduced ejection fraction and in control group and assess its potential prognostic value. 


\section{Materials \& method Patients}

The study group consisted of 130 consecutive patients admitted to the IInd Clinic of Cardiology in the University Hospital no 2 in Bydgoszcz (Poland) with the principal diagnosis of chronic heart failure. In $65(50 \%)$ of them the main reason for admittance was the exacerbation of heart failure symptoms. The other half of patients was admitted electively for device implantation or periodic assessment of the disease progression (patient on the elective heart transplant list). All patients underwent careful anamnesis and physical examination. Presence of peripheral edema or pulmonary congestion defined as any crackles or rales (classical Killip II class) was recorded by the admitting physician. The inclusion criteria consisted of: chronic heart failure diagnosed according to European Society of Cardiology Guidelines on Acute and Chronic Heart Failure Diagnosis and Treatment and left ventricular ejection fraction $\leq 45 \%$ assessed during index hospitalization or during past 6 months. All patients before entering the study must have received optimal pharmacologic treatment for at least 3 months. Individuals admitted with acute heart failure defined as cardiogenic shock or pulmonary edema, were excluded from the study. Patients diagnosed with acute coronary syndrome, active infection or neoplastic disease, fever of unknown origin, anemia requiring iron supplementation, chronic obstructive pulmonary disease, autoimmune disorders and other chronic inflammatory diseases, decompensated diabetes requiring intravenous insulin infusions or severely compromised renal function with glomerular filtration rate below $30 \mathrm{ml} / \mathrm{h}$ were also excluded from the study. The demographic and clinical features of the study group are summarized in Table 1.

\section{Control group}

The control group consisted of 32 healthy adults matched for age and sex. They were recruited from

Table 2. Comparison of melanoma cell adhesion molecule and NT-proBNP plasma concentration in patients with chronic heart failure and controls together with relations to the clinical status.

\begin{tabular}{|c|c|c|c|c|c|c|}
\hline Group & Minimum & $\begin{array}{l}\text { Lower } \\
\text { quartile }\end{array}$ & Median & $\begin{array}{l}\text { Upper } \\
\text { quartile }\end{array}$ & Maximum & p-value \\
\hline \multicolumn{7}{|l|}{ MCAM (ng/ml) } \\
\hline Control & 37.24 & 199.34 & 348.25 & 555.14 & 958.00 & $0.0103^{+}$ \\
\hline CHF all & 31.56 & 267.27 & 504.80 & 834.53 & 4432.35 & $0.9940^{\ddagger}$ \\
\hline CHF stable & 31.56 & 265.05 & 466.74 & 815.02 & 3149.8 & - \\
\hline CHF exacerbation & 63.22 & 270.32 & 522.20 & 838.91 & 4432.35 & - \\
\hline \multicolumn{7}{|l|}{ NT-proBNP (pg/ml) } \\
\hline CHF all & 60 & 711 & 1862 & 3938 & 51,636 & $<0.0001^{\ddagger}$ \\
\hline CHF stable & 60 & 429 & 1051 & 2179 & 9158 & - \\
\hline CHF exacerbation & 186 & 1387 & 3235 & 5461 & 51,636 & - \\
\hline \multicolumn{7}{|l|}{ MCAM (ng/ml) } \\
\hline Edema & 31.56 & 341.00 & 515.40 & 823.65 & 3536.05 & 0.5026 \\
\hline No edema & 63.22 & 257.17 & 481.81 & 823.99 & 4432.35 & - \\
\hline \multicolumn{7}{|l|}{ NT-proBNP (ng/ml) } \\
\hline Edema & 186 & 1269 & 3210 & 7369 & 51646 & $<0.0001$ \\
\hline No edema & 60 & 569 & 1307 & 3252 & 16,125 & - \\
\hline \multicolumn{7}{|l|}{ MCAM (ng/ml) } \\
\hline Congestion & 31.56 & 324.42 & 464.44 & 706.83 & 3536.05 & 0.7811 \\
\hline No congestion & 63.22 & 265.16 & 534.00 & 878.20 & 4432.35 & - \\
\hline \multicolumn{7}{|l|}{ NT-proBNP (pg/ml) } \\
\hline Congestion & 152 & 1035.5 & 2943 & 6160 & 51,646 & 0.0088 \\
\hline No congestion & 60 & 645.5 & 1409 & 3774.2 & 16,125 & - \\
\hline
\end{tabular}


the Cardiology Outpatient Department after careful exclusion of all cardiovascular diseases and those listed above in the exclusion criteria.

Written informed consent was obtained from each study participant following a detailed explanation of the study protocol that conforms to the principles outlined in the Declaration of Helsinki. Research Ethical Committee by the Collegium Medicum of the Nicolaus Copernicus University approved the study protocol.

\section{Laboratory tests}

On the first day of hospitalization, all enrolled patients have blood sampling for routine laboratory test including complete blood count, blood electrolytes, creatinine, troponin $\mathrm{T}(\mathrm{TnT}), \mathrm{NT}$-proBNP and C-reactive protein (CRP) concentration. At the same time blood samples for determination of MCAM plasma concentration were taken. Fasting blood samples were collected between 7 and 8 a.m., in the supine position; the venopuncture was done in the antecubital region, with minimal venostasis. The samples were collected into polyetylen Vacutainer ${ }^{\circledR}$ (Beckton Dickinson, NJ, USA) test tubes containing anticoagulant $-3.2 \%$ sodium citrate and to test tubes without anticoagulant. The blood samples for MCAM determination in tubes containing anticoagulant were then centrifuged at $3000 \times \mathrm{g}$ at $4^{\circ} \mathrm{C}$ for $10 \mathrm{~min}$, thus the obtained low-platelet concentration plasma was sampled into Eppendorf test tubes and frozen at $-80^{\circ} \mathrm{C}$ for a period of maximum 3 months before the measurement was taken. Plasma samples of controls were prepared in the same way as from chronic heart failure (CHF) patients. No repeated freeze-thaw cycles were preformed before analysis.

The remaining tests were immediately performed in the University Hospital nr 2 in Bydgoszcz local laboratory. TnT concentration was determined with the use of sandwich electrochemiluminescence immunoassay (Elecsys ${ }^{\circledR}$ Troponin $\mathrm{T}$ high sensitivity assay, Roche Diagnositcs GmbH, Mannheim, Germany). NT-proBNP plasma level was determined by means of sandwich chemiluminescence immunoassay (Elecsys ${ }^{\circledR}$ proBII NP, Roche Diagnositcs GmbH) and CRP concentration was measured with the use of high sensitivity immunoturbidimetric method (CRP OSR6199 Highly Sensitive Application, Olympus America, NY, USA.)

MCAM plasma concentration was determined in the Department of Immunology of Collegium Medicum of Nicolaus Copernicus University with the use of commercially available, highly sensitive enzymelinked immunosorbent assay (E91670Hu, USCN Life Science, Wuhan, China) strictly according to the manufacturer instructions.

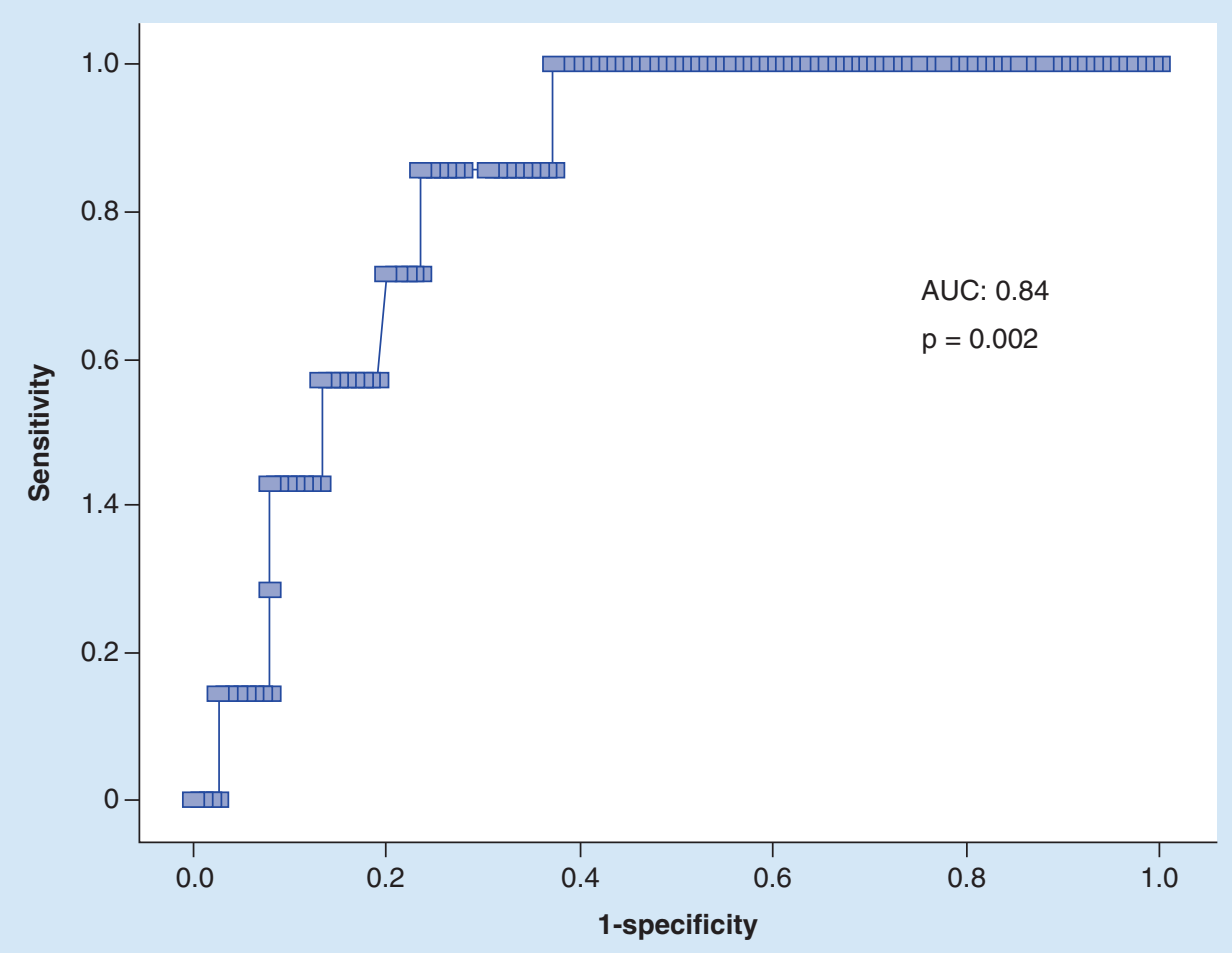

Figure 1. Receiver operator curve analysis of melanoma cell adhesion molecule plasma level as a predictor of death during 12-month follow-up.

AUC: Area under the curve. 


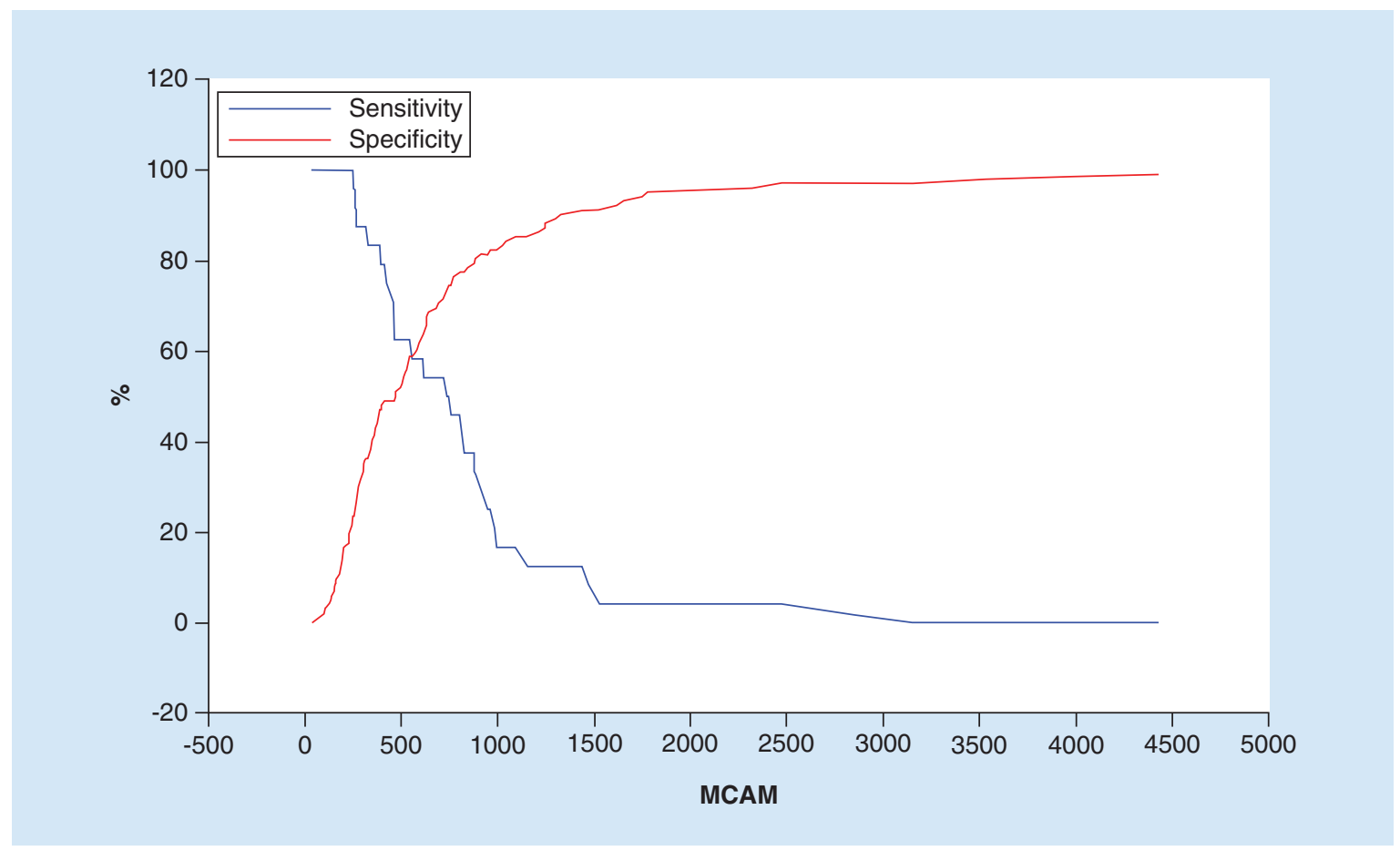

Figure 2. Specificity and sensitivity of MCAM plasma concentration $500 \mathrm{ng} / \mathrm{ml}$ - chosen cutoff value.

MCAM: Melanoma cell adhesion molecule.

Follow-up

Telephone follow-up visits were performed every 3 months for 1 year. End points of the study were: death from all causes and unplanned hospitalization for heart failure worsening.

\section{Statistical method}

Statistical analyses were preformed with the use of PQStat 1.4.8 software. Continuous variables with normal distribution are expressed as means with standard deviations. Variables with skewed distribution are presented as medians and lower and upper quartiles. Receiver operator characteristic curves (ROC) were created to investigate the diagnostic and prognostic value of MCAM plasma level. Kaplan-Meier plots were constructed to study the impact of elevated MCAM concentration on overall survival. The relationship between MCAM plasma concentration and prespecified end points was analyzed with the use of multivariate logistic regression model. Kruskal-Wallis test with post hoc Bonferroni correction was used to assess the difference of the studied parameters between controls and stable and exacerbated CHF patients. U-Mann-Whitney test was used to analyze differences in MCAM plasma level between patients with or without pulmonary congestion or peripheral edema. Relationship between studied parameters was evaluated with Spearman rank correlation coefficient. A probability less than 0.05 was considered statistically significant.

\section{Results}

Out of 130 patients included in the analysis 65 were admitted to the hospital because of chronic heart failure exacerbation. Ischemic etiology of CHF was evident in about half of the investigated group. Mean age of patients was 60 years and the majority $(83 \%)$ were men. Mean body mass index was elevated and 36\% of patients suffered from diabetes mellitus. Both mean hemoglobin value and mean corpuscular volume were in normal range. CRP plasma level and TnT concentration were only slightly elevated. As expected, mean NT-proBNP plasma level was markedly elevated in the study group. Pharmacologic treatment was consistent with the guidelines of the European Society of Cardiology and almost all patients received angiotensin converting enzyme inhibitors (ACEI) or angiotensin receptor blockers (ARB) and betablockers (BB). Fourteen (10\%) patients died during 1-year follow-up. Sixty unplanned hospitalizations with heart failure exacerbation were observed during follow-up period. Clinical and laboratory characteristics of the study group are summarized in Table 1.

MCAM plasma level was significantly higher in the study than in control group (504 vs $348 \mathrm{ng} / \mathrm{ml}$, $\mathrm{p}=0.01$ ). ROC analysis revealed that MCAM may differentiate patients with and without heart failure (area under the curve [AUC]: 0.74; 95\% CI: 0.69-0.79; $\mathrm{p}=0.01$ ). Neither exacerbation of heart failure nor the presence of peripheral edema or pulmonary congestion 
had the influence of MCAM plasma concentration (Table 2).

ROC analysis was performed to assess the value of MCAM plasma concentration in predicting end points of the study. It revealed that MCAM might serve as a predictor of death during 12-month follow-up (AUC: 0.8404; $\mathrm{p}<0.002$; Figure 1).

Cutoff value suggested by ROC analysis was 398 $\mathrm{ng} / \mathrm{ml}$, however it has been decided to select the cutoff of $500 \mathrm{ng} / \mathrm{ml}$, which was the median concentration in the CHF group. Sensitivity and specificity for the selected cutoff are presented in Figure 2.

Survival analysis with Kaplan-Meier plots constructed for a cutoff point fixed at $500 \mathrm{ng} / \mathrm{ml}$ showed that patients with MCAM above $500 \mathrm{ng} / \mathrm{ml}$ had almost $10 \%$ lower probability of survival than individuals with MCAM <500 ng/ml (Figure 3).

After adjustment for age, left ventricular ejection fraction, NYHA functional class, hemoglobin level, NT-proBNP, CRP, TnT plasma concentration and glomerular filtration rate, multivariate Cox regression model did not confirm the value of MCAM as an independent predictor of worse prognosis in patients with chronic heart failure. Among established prognostic factors only age and NT-proBNP turned out to be the independent predictor of death in 12-month observation (Table 3).
MCAM plasma concentration did not allow for prediction of hospitalization with heart failure worsening during one-year follow-up. MCAM plasma concentration significantly $(\mathrm{p}=0.0156)$ weakly, negatively correlated $(R=-0.2160)$ with left ventricular ejection fraction and positively $(r=0.2268 ; \mathrm{p}=0.0116)$ with NT-proBNP plasma concentration.

\section{Discussion}

Efficient invasive treatment of acute coronary syndromes saved millions of lives throughout the world, however significant portion of these patients has been left with the impairment of left ventricular systolic function, ultimately leading to chronic heart failure. Increasing $\mathrm{CHF}$ morbidity with its enormous economic burden is the most important challenge of modern cardiology. Clinical characteristic of this complex syndrome is inextricably associated with frequent relapse of symptoms and the need for rehospitalization. It seems to be the main reason of constant search for reliable markers of worse prognosis allowing for early selection of patients requiring closer follow-up and more aggressive treatment including, in some cases, left ventricular assist devices.

In the present study we made an attempt to assess diagnostic and prognostic value of MCAM - a novel surrogate marker of endothelial damage.

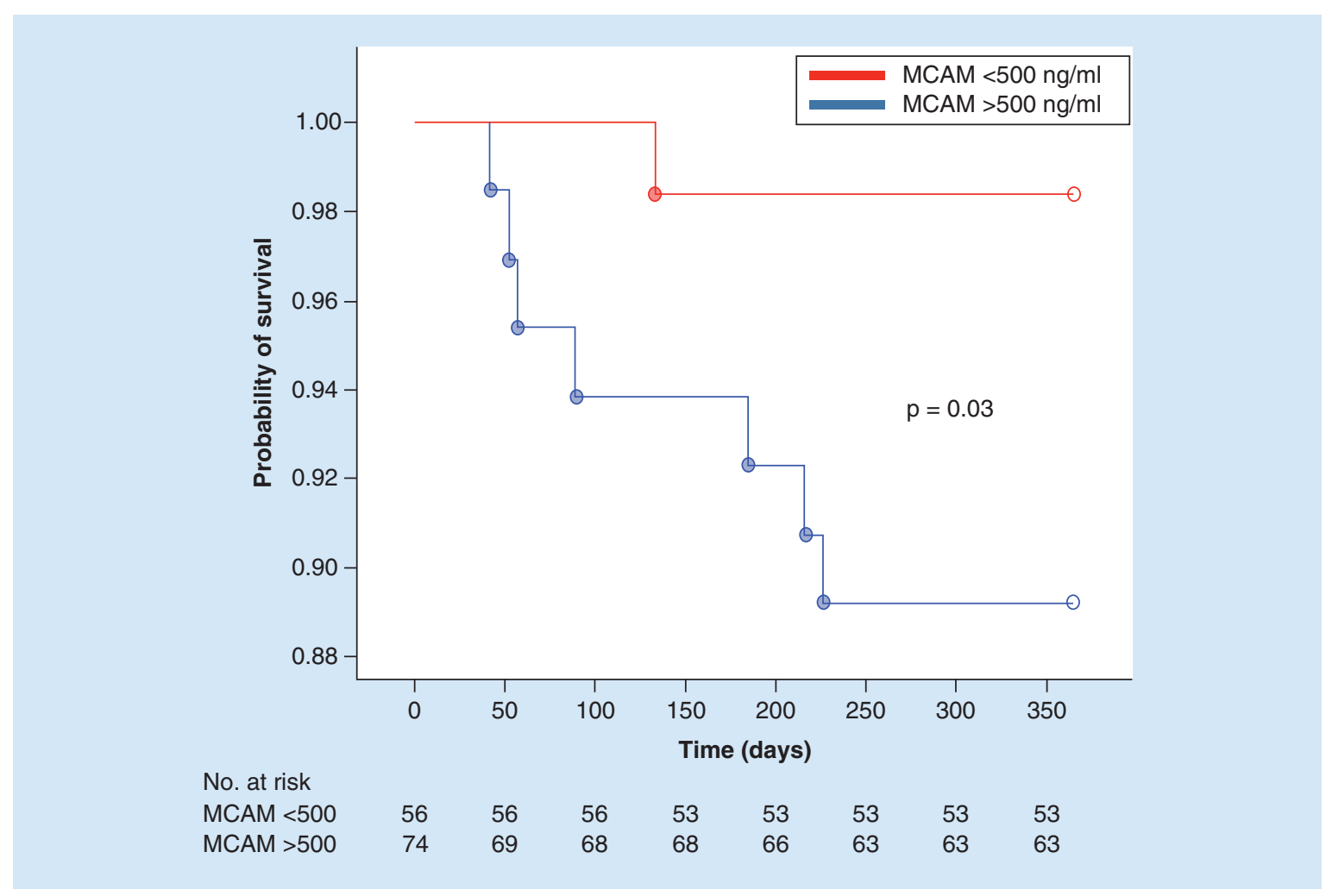

Figure 3. Kaplan-Meyer survival analysis according to melanoma cell adhesion molecule plasma concentration. MCAM: Melanoma cell adhesion molecule 
Table 3. Multivariate Cox regression analysis of all-cause mortality.

\begin{tabular}{|lllll|}
\hline Factor & p-value & HR & $-95 \% \mathbf{C l}$ & $+95 \% \mathbf{C l}$ \\
\hline MCAM $(\mathrm{ng} / \mathrm{ml})$ & 0.784446 & 1.000081 & 0.99 & 1.00 \\
\hline LVEF $(\%)$ & 0.491923 & 0.982584 & 0.93 & 1.03 \\
\hline Age (years) & 0.027 & 1.038 & 1.004 & 1.074 \\
\hline NYHA class & 0.055099 & 0.237275 & 0.05 & 1.03 \\
\hline NTproBNP $(\mathrm{pg} / \mathrm{ml})$ & 0.002898 & 1.000056 & 1.000019 & 1.000093 \\
\hline CRP $(\mathrm{mg} / \mathrm{l})$ & 0.172 & 1.029 & 0.988 & 1.072 \\
\hline TnT $(\mu \mathrm{g} / \mathrm{l})$ & 0.905 & 1.815 & 0.000 & 30,864 \\
\hline GFR $(\mathrm{ml} / \mathrm{h})$ & 0.135 & 1.043 & 0.987 & 1.103 \\
\hline Hb $(\mathrm{g} / \mathrm{dl})$ & 0.425041 & 0.894166 & 0.93 & 1.03 \\
\hline $\begin{array}{l}\text { GFR: Glomerular filtration rate; Hb: Hemoglobin; HR: Hazard ratio; LVEF: Left ventricular ejection fraction; MCAM: Melanoma cell adhesion } \\
\text { molecule; NYHA: New York Heart Association functional class. }\end{array}$ & & & \\
\hline
\end{tabular}

We decided to assess endothelial dysfunction using MCAM plasma concentration considering its clinical availability and technical simplicity comparing to direct evaluation of CEC number. Furstenberg et al. described a stable expression of MCAM on CEC demonstrated in the flow cytometric measurement, which led to the assumption that MCAM mRNA quantification would reflect CEC amount in peripheral blood. Real-time-PCR measurement of MCAM mRNA revealed that it is in fact equivalent to CEC quantification and allows for reliable and easy assessment of endothelial status [9]. These findings suggest that simple and cost-effective ELISA method used for MCAM measurement can be a promising alternative to complex and expensive CEC determination. MCAM may also be useful in identification of very early, not yet clinically evident endothelial dysfunction. Dogansen et al. demonstrated elevated MCAM plasma concentration, measured by ELI method $\mathrm{SA}$, in young patients with Type 1 diabetes mellitus without diabetes-related complication and other cardiovascular risk factors. Researchers suggest that MCAM may be a better marker of early endothelial dysfunction than carotid intima-media thickness [14].

The results of the presented study show that MCAM plasma concentration is, as we expected, higher in patients with chronic heart failure with reduced ejection fraction than in controls. This finding is consistent with previous observation by Chong et al. indicating increased numbers of circulating endothelial cells in patients with stable chronic heart failure. In that paper quantification of CEC was chosen as the most direct indicator of severe endothelial disruption and observed their expected inverse correlation with flow-mediated dilation, which in researchers' opinion confirms that endothelial dysfunction is generalized phenomenon in heart failure. Authors suggest that the impairment of physiological function, as indicated by decreased Findi- ces $\mathrm{MD}$, together with elevated CEC numbers point out toward extensive endothelial disarrangement, which may even involve denudation of some parts of vascular bed [12]. This kind of damage associated with the exposure of prothrombotic subendothelial layer can provide mechanistic explanation for the observed increased thromboembolic risk in patients with advanced heart failure [15]. Yet, the prognostic value of flow-mediated dilation impairment and increased CEC was not investigated. Another paper by Chong et al. assessing endothelial damage through CEC quantification covered also population of patients with acute heart failure. Again, it was observed that the amount of circulating endothelial cells increases in heart failure, but this time authors revealed that clinical stability had no impact on this phenomenon as both AHF and CHF patients had elevated CEC number [13]. Martínez-Sales et al. also observed increased amount of CEC in patients with heart failure, however in their group of patients the elevation was more pronounced in the acute phase of the disease. Unfortunately it is not precisely stated whether it was AHF or CHF exacerbation [16]. None of the above mentioned researchers investigated the prognostic significance of CEC number elevation. In our study we demonstrated increased concentration of MCAM in plasma of patients with heart failure, however we could not demonstrate the difference between patients admitted for heart failure exacerbation and those in stable condition. Moreover, there was no association between clinical findings consistent with heart failure exacerbation and MCAM. Analysis of baseline characteristic of Martínez-Sales et al. study population may shed some light on this divergent results - only about half of investigated heart failure patients at baseline received betablockers and two-thirds took ACEI/ARB, whereas in our population fairly all patients were treated with both agents. Vasoprotection and the improvement of endothelial function are well known effects of both: 
angiotensin-converting enzyme inhibitors [17] and betablockers [18], thus it seems possible that the wide use of these agents in our study may diminish the impact of acute state on endothelial dysfunction reflected by MCAM concentration. However, it may also indicate that in some clinical conditions soluble form of MCAM measured in our research does not precisely reflect circulating endothelial cells amount.

The only one paper covering the topic of MCAM plasma level and heart failure was published recently by Gayat et al. in International Journal of Cardiology. Authors measured MCAM concentration in patients admitted to the emergency department with acute dyspnea and assess the diagnostic accuracy of MCAM in distinguishing patients with AHF-related symptoms. It was observed that patients with final diagnosis of acute heart failure had significantly higher MCAM concentration than individuals with different etiology of dyspnea. Moreover, combination of MCAM and NT-proBNP further improved diagnostic accuracy and allowed for identification of more patients with AHF than NT-proBNP alone [19]. Gayat et al. investigated a total of 391 patients with acute symptoms and tried to associate clinical findings with that of experimental studies, however they did not attempt to assess the prognostic value of MCAM concentration. To the best of our knowledge our work is the only one investigating the influence of MCAM plasma level on long-term prognosis of patients with chronic heart failure with reduced ejection fraction. We proved that patients with MCAM concentration higher that median value observed in our study have worse prognosis than those with concentration below $500 \mathrm{ng} / \mathrm{ml}$. Endothelial dysfunction is known to unfavorably influence prognosis in chronic heart failure. Fischer $e t a l$. observed higher mortality and morbidity in CHF patients with impaired flow-dependent endothelial mediated vasodilatation and proved that in this population endothelial damage reflected by the decrease in FDD indices is an independent predictor of adverse outcome including death, hospitalization for heart failure worsening or heart transplant [20]. In our study we chose two separate primary end points - hospitalization for heart failure worsening and death of all causes. We did not demonstrate the influence of MCAM concentration on the rate of hospitalization for CHF exacerbation. ROC analysis used in our study revealed that MCAM might be a valuable predictor of death (AUC: 0.8404; $\mathrm{p}<0.002$ ) in CHF patients during medium-term follow-up. However, multivariate Cox regression model did not confirm the value of MCAM as an independent predictor of worse prognosis in patients with chronic heart failure. The reasons behind this finding might include too small study group or not sufficient number of events during one-year follow-up. In our analysis only NT-proBNP and age turned out to be the independent predictors of death in 12-month observation, which is not surprising, as natriuretic peptides are one of the most powerful predictors of adverse outcome. NT-proBNP is a marker closely associated with patient's hemodynamic status thus reflecting precisely the severity of heart failure and remaining in strong relation to clinical signs and symptoms. Endothelial dysfunction resulting in MCAM increase is a very important process in numerous diseases but its role in chronic heart failure might not be so prominent. In our study mean left ventricular ejection fraction was about $27 \%$ and majority of patients were in NYHA functional class III. Such a deep hemodynamic compromise might diminish the influence of endothelial dysfunction on prognosis accentuating the prognostic value of markers associated with hemodynamic overload - namely natriuretic peptides. Nevertheless, MCAM as marker reflecting endothelial status is worth further investigation in both CHF and other cardiovascular disease, with special regard to syndromes traditionally involving endothelial dysfunction like coronary artery disease or arterial hypertension.

\section{Conclusion}

MCAM for the first time was shown to increase in patients with chronic heart failure, both in exacerbation and in stable form of the disease. Strong relation of MCAM and circulating endothelial cells - an established marker of endothelial damage - indicates the presence of endothelial disruption associated with heart failure. Moreover, MCAM plasma concentration seems to be a promising marker of worse prognosis in $\mathrm{CHF}$ with reduced ejection fraction. Considering its simplicity and availability of ELISA method, MCAM may be a valuable supplement in multimarker approach to heart failure patients. Further research is needed to formulate best configuration of markers facilitating the choice of optimal treatment strategy for this growing population of cardiovascular patients.

\section{Financial \& competing interests disclosure}

This research was supported by the Collegium Medicum of Nicolaus Copernicus University grant no. 830. The authors have no other relevant affiliations or financial involvement with any organization or entity with a financial interest in or financial conflict with the subject matter or materials discussed in the manuscript apart from those disclosed.

No writing assistance was utilized in the production of this manuscript.

\section{Ethical conduct of research}

The authors state that they have obtained appropriate institutional review board approval or have followed the principles 
outlined in the Declaration of Helsinki for all human or animal experimental investigations. In addition, for investigations involving human subjects, informed consent has been obtained from the participants involved.

\section{Open access}

This work is licensed under the Attribution-NonCommercialNoDerivatives 4.0 Unported License. To view a copy of this license, visit http://creativecommons.org/licenses/by-nc-nd/4.0/

\section{Executive summary}

- Melanoma cell adhesion molecule (MCAM) is a marker of endothelial damage related to the amount of circulating endothelial cells (CEC).

- Endothelial damage was recorded in numerous cardiovascular conditions including heart failure.

- Determination of MCAM plasma concentration is easier and cheaper than CEC quantification and could be an interesting alternative method of endothelial damage assessment.

- MCAM plasma concentration has never been assessed in patients with chronic heart failure.

- The aim of the study was to evaluate the diagnostic and prognostic significance in hospitalized patients with heart failure with reduced ejection fraction.

\section{Materials \& method}

- 130 patients with heart failure and left ventricular ejection fraction $\leq 45 \%$ admitted to Clinic of Cardiology. Half of them were admitted with exacerbation of heart failure symptoms and the other half were stable admitted for elective reasons.

- Control group consisted of 32 healthy volunteers after careful exclusion of cardiovascular disorders.

- Anamnesis, physical examination, routine laboratory test and blood sampling for MCAM plasma determination were performed.

- Telephone follow-up visits performed every 3 months for one year, assessed study end points: death of all causes and hospitalization for heart failure exacerbation.

Results

- MCAM plasma level was significantly higher in the study than in control group (504 vs $348 \mathrm{ng} / \mathrm{ml} ; \mathrm{p}=0.01$ ).

- Neither exacerbation of heart failure, nor the presence of peripheral edema or pulmonary congestion had the influence of MCAM plasma concentration.

- Receiver operator curve analysis of survival indicates that MCAM may be a valuable predictor of all cause mortality in heart failure patients during 12-month observation (AUC: $0.8404 ; p<0.002$ ).

- Patients with MCAM above $500 \mathrm{ng} / \mathrm{ml}$ had almost $10 \%$ lower probability of survival than individuals with MCAM $<500 \mathrm{ng} / \mathrm{ml}(\mathrm{p}=0.03)$.

- In multivariate analysis only age and NT-proBNP plasma level were independent predictors of worse prognosis. Discussion

- According to available data MCAM may be considered equivalent to more complicated and expensive CEC quantification.

- CEC number was previously proved to increase in heart failure suggesting the presence of endothelial dysfunction in this condition.

- Prognostic significance of endothelial damage evaluated by means of CEC determination has never been assessed in heart failure patients, however flow mediated dilation impairment was associated with worse prognosis.

- The only available study of MCAM in heart failure patients showed increased concentration of the parameter during acute episode of heart failure in the emergency settings.

- Prognostic significance of MCAM plasma concentration has not been assessed before in heart failure.

Conclusion

- MCAM plasma concentration, being a surrogate marker of endothelial damage is elevated in patients with chronic heart failure with reduced ejection fraction.

- Apart from plausible diagnostic utility, MCAM is a promising prognostic factor in predicting all-cause mortality in chronic heart failure population.

\section{References}

Papers of special note have been highlighted as:

- of interest; $\bullet$ of considerable interest

1 Lehmann JM, Holzmann B, Breitbart EW, Schmiegelow P, Riethmuller G, Johnson JP. Discrimination between benign and malignant cells of melanocytic lineage by two novel antigens, a glycoprotein with a molecular weight of 113,000 and a protein with a molecular weight of 76,000. Cancer Res. 47, 841-845 (1987).
2 Wu GJ, Wu MW, Wang SW et al. Isolation and characterization of the major form of human $M U C 18$ cDNA gene and correlation of MUC18 over-expression in prostate cancer cell lines and tissues with malignant progression. Gene. 1, 17-31 (2001).

3 Witze ES, Litman ES, Argast GM, Moon RT, Ahn NG. Wnt5a control of cell polarity and directional movement by polarized redistribution of adhesion receptors. Science 5874 , 365-369 (2008). 
4 Bardin N, Anfosso F, Masse JM et al. Identification of CD146 as a component of the endothelial junction involved in the control of cell-cell cohesion. Blood 98, 3677-3684 (2001).

5 Shih Ie-Ming. The role of CD146 (Mel-CAM) in biology and pathology. J. Pathol. 189, 4-11 (1999).

6 Clancy R, Marder G, Martin V, Belmont HM, Abramson $\mathrm{SB}$, Buyon J. Circulating activated endothelial cells in systemic lupus erythematosus: further evidence for diffuse vasculopathy. Arthritis Rheum. 44, 1203-1208 (2001).

7 Camoin-Jau L, Kone-Paut I, Chabrol B, Sampol J, DignatGeorge F. Circulating endothelial cells in Behcet's disease with cerebral thrombophlebitis. Thromb. Haemost. 83, 631-632 (2000).

8 Mutunga M, Fulton B, Bullock R et al. Circulating endothelial cells in patients with septic shock. Am. J. Respir. Crit. Care Med. 163, 195-200 (2001).

9 Furstenberger G, Moos R von, Senn HJ, Boneberg EM. Real-time PCR of CD146 mRNA in peripheral blood enables the relative quantification of circulating endothelial cells and is an indicator of angiogenesis. Br. J. Cancer 93, 793-798 (2005).

- The research proves that melanoma cell adhesion molecule determination may be a reasonable alternative to circulating endothelial cells (CECs) quantification.

10 Ozdogu H, Sozer O, Boga C, Kozanoglu I, Mytalman E, Guzey M. Flow cytometric evaluation of circulating endothelial cells: W new protocol for identifying endothelial cells at several stages of differentiation. Am. J. Hematol. 82, 706-711 (2007).

11 Blann AD, Woywodt A, Bertolini F et al. Circulating endothelial cells. Biomarker of vascular disease. Thromb. Haemost. 93, 228-235 (2005).

- The paper summarizing the significance of CECs in cardiovascular disorders.

12 Chong AY, Blann AD, Patel J, Freestone B, Hughes E, Lip GY. Endothelial dysfunction and damage in congestive heart failure: relation of flow-mediated dilation to circulating endothelial cells, plasma indexes of endothelial damage, and brain natriuretic peptide. Circulation 110, 1794-1798 (2004).

- The study confirming that endothelial damage assessed with various methods is actually present in heart failure patients.
13 Chong AY, Lip GY, Freestone B, Blann AD. Increased circulating endothelial cells in acute heart failure: comparison with von Willebrand factor and soluble E-selectin. Eur. J. Heart Fail. 8, 167-172 (2006).

14 Ciftci Dogansen S, Helvaci A, Adas M, Deniz Onal S. The relationship between early atherosclerosis and endothelial dysfunction in Type 1 diabetic patients as evidenced by measurement of carotid intima-media thickness and soluble CD146 levels: a cross sectional study. Cardiovasc. Diabetol. 12, 153 (2013).

15 de Peuter OR, Kok WE, Torp-Pedersen C, Büller HR, Kamphuisen PW. Systolic heart failure: a prothrombotic state. Semin. Thromb. Hemost. 35, 497-504 (2009).

16 Martínez-Sales V, Sánchez-Lázaro I, Vila V, Almenar L, Contreras T, Reganon E. Circulating endothelial cells in patients with heart failure and left ventricular dysfunction. Dis. Markers 31, 75-82 (2011).

- The paper confirming endothelial damage with the use of CECs quantification in heart failure patients with reduced ejection fraction.

17 Pepine CJ. Improved endothelial function with angiotensinconverting enzyme inhibitors. Am. J. Cardiol. 79, 29-32 (1997).

18 Peller M, Ozierański K, Balsam P, Grabowski M, Filipiak KJ, Opolski G. Influence of beta-blockers on endothelial function: a meta-analysis of randomized controlled trials. Cardiol. J. 22(6), 708-716 (2015).

19 Gayat E, Caillard A, Laribi S et al. Soluble CD146, a new endothelial biomarker of acutely decompensated heart failure. Int. J. Cardiol. 199, 241-247 (2015).

-• The only one paper on melanoma cell adhesion molecule plasma concentration in patients during the acute episode of heart failure.

20 Fischer D, Rossa S, Landmesser U et al. Endothelial dysfunction in patients with chronic heart failure is independently associated with increased incidence of hospitalization, cardiac transplantation, or death. Eur. Heart J. 26, 65-69 (2005). 